\title{
Data Cutoff Date Description
}

National Cancer Institute

\section{Source}

National Cancer Institute. Data Cutoff Date Description. NCI Thesaurus. Code C98718.

A textual description of the data cutoff date. 